\title{
CT-to-fluoroscopy registration versus scan-and-plan registration for robot-assisted insertion of lumbar pedicle screws
}

\author{
Asham Khan, MD, ${ }^{1,2}$ Mohamed A. R. Soliman, MD, MSc, PhD, ${ }^{1-3}$ Nathan J. Lee, MD, ${ }^{4}$ \\ Muhammad Waqas, MBBS, ${ }^{1,2}$ Joseph M. Lombardi, MD, ${ }^{4}$ Venkat Boddapati, MD, ${ }^{4}$ \\ Lauren C. Levy, ${ }^{2}$ Jennifer Z. Mao, MBA, ${ }^{1,2}$ Paul J. Park, MD, ${ }^{4}$ Justin Mathew, MD, ${ }^{4}$ \\ Ronald A. Lehman Jr., MD, ${ }^{4}$ Jeffrey P. Mullin, MD, MBA, ${ }^{1,2}$ and John Pollina, MD ${ }^{1,2}$ \\ 'Department of Neurosurgery, Jacobs School of Medicine and Biomedical Sciences, University at Buffalo; ${ }^{2}$ Department \\ of Neurosurgery, Buffalo General Medical Center, Kaleida Health, Buffalo, New York; ${ }^{3}$ Department of Neurosurgery, Cairo \\ University, Cairo, Egypt; and ${ }^{4}$ Department of Orthopedic Surgery, Columbia University Medical Center, The Och Spine Hospital \\ at NewYork-Presbyterian, New York, New York
}

OBJECTIVE Pedicle screw insertion for stabilization after lumbar fusion surgery is commonly performed by spine surgeons. With the advent of navigation technology, the accuracy of pedicle screw insertion has increased. Robotic guidance has revolutionized the placement of pedicle screws with 2 distinct radiographic registration methods, the scanand-plan method and CT-to-fluoroscopy method. In this study, the authors aimed to compare the accuracy and safety of these methods.

METHODS A retrospective chart review was conducted at 2 centers to obtain operative data for consecutive patients who underwent robot-assisted lumbar pedicle screw placement. The newest robotic platform (Mazor X Robotic System) was used in all cases. One center used the scan-and-plan registration method, and the other used CT-to-fluoroscopy for registration. Screw accuracy was determined by applying the Gertzbein-Robbins scale. Fluoroscopic exposure times were collected from radiology reports.

RESULTS Overall, 268 patients underwent pedicle screw insertion, 126 patients with scan-and-plan registration and 142 with CT-to-fluoroscopy registration. In the scan-and-plan cohort, 450 screws were inserted across 266 spinal levels (mean $1.7 \pm 1.1$ screws/level), with 446 (99.1\%) screws classified as Gertzbein-Robbins grade A (within the pedicle) and $4(0.9 \%)$ as grade B (<2-mm deviation). In the CT-to-fluoroscopy cohort, 574 screws were inserted across 280 lumbar spinal levels (mean $2.05 \pm 1.7$ screws/ level), with $563(98.1 \%)$ grade A screws and $11(1.9 \%)$ grade $B(p=0.17)$. The scan-and-plan cohort had nonsignificantly less fluoroscopic exposure per screw than the CT-to-fluoroscopy cohort (12 \pm 13 seconds vs $11.1 \pm 7$ seconds, $p=0.3$ ).

CONCLUSIONS Both scan-and-plan registration and CT-to-fluoroscopy registration methods were safe, accurate, and had similar fluoroscopy time exposure overall.

https://thejns.org/doi/abs/10.3171/2021.10.FOCUS21506

KEYWORDS robotic spine surgery; pedicular screws; scan-and-plan; CT-to-fluoroscopy; navigation

$\mathrm{M}$ ISPLACED pedicle screws during spine surgery can lead to neurological and vascular compromise after spinal fusion surgery., ${ }^{1,2}$ To decrease adverse outcomes associated with misplaced screws, surgeons have adopted various navigation technologies to increase the accuracy of screw insertion, including fluoroscopy-assisted, CT-guided, and, recently, robot-assisted technologies. $^{3-6}$ These technologies have been shown to decrease the risk of misplaced screws and complications associated with misplaced screws. Robot-assisted pedicle screw insertion has demonstrated reproducible accuracy as it pertains to pedicle screw insertion via the traditional trajectory and the cortical bone screw trajectories. ${ }^{6-8}$

Robotic technology, as it pertains to spine surgery, has 2 distinct registration methods: CT-to-fluoroscopy registration and scan-and-plan registration. ${ }^{7}$ Both registration 
methods use image-guided software to plan the trajectories for pedicle screw placement. With CT-to-fluoroscopy registration, a CT scan is obtained preoperatively and mapped with anteroposterior and lateral intraoperative fluoroscopic radiographs to plan the trajectories. With scan-and-plan registration, a CT scan is obtained using an $\mathrm{O}$-arm (O-arm Surgical Imaging System, Medtronic) while the patient is positioned on the operating room table, and a surgical plan with pedicle screw trajectories is computed by the robot software (Mazor Robotics Ltd.). A previous study ${ }^{7}$ compared a small sample of 8 patients in whom the CT-to-fluoroscopy registration method was used with 12 patients in whom the scan-and-plan technique was used and demonstrated no difference in the accuracy of pedicle screw placement. In the current study, we aimed to compare accuracy (Gertzbein-Robbins scale) and safety (radiation exposure and complications) among a larger cohort of patients in whom the 2 different registration techniques were applied at 2 centers. To our knowledge, this is the first comprehensive study to directly compare these radiographic registration techniques.

\section{Methods \\ Ethical Considerations}

The University at Buffalo and Columbia University IRBs reviewed the protocol for this study, and it was given an exempt determination by both. The Strengthening the Reporting of Observational Studies in Epidemiology (STROBE) reporting guidelines were followed. ${ }^{9}$

The scan-and-plan registration method was utilized for patients who underwent surgical intervention at the University at Buffalo, while the CT-to-fluoroscopy registration method was utilized for patients who underwent surgical intervention at Columbia University. Informed consent was obtained from all patients who underwent surgical intervention.

\section{Study Design and Population}

We conducted a retrospective review of data for consecutive patients who underwent robot-assisted lumbar pedicle screw insertion using the Mazor X Robotic System (Medtronic) between May 2017 and December 2019. The scan-and-plan registration method was utilized for patients who underwent surgical intervention at one center, while the CT-to-fluoroscopy registration method was utilized for those who underwent surgical intervention at the other center. All operations at the center using scan-and-plan registration were performed by a single neurosurgeon (J.P.) with assistance from neurosurgical residents. All operations at the CT-to-fluoroscopy center were performed by a single orthopedic surgeon (R.A.L.) with assistance from orthopedic surgery residents. Both surgeons began using the robot at the same time, so they had similar experience with its use.

\section{Data Collection}

Data collected included patient demographics (e.g., age, sex, BMI, smoking history, comorbidities, and American Society of Anesthesiologists [ASA] physical status classification), diagnosis, operation duration (skin-to-skin time), fluoroscopy duration (during the whole procedure), spinal level, and intraoperative blood loss.

Pedicle screw position was graded as described by the Gertzbein-Robbins scale..$^{10}$ If the screw was completely within the pedicle, it was grade $\mathrm{A}$; a $<2-\mathrm{mm}$ deviation was grade $\mathrm{B}$; a $2-\mathrm{mm}$ to $4-\mathrm{mm}$ deviation was grade $\mathrm{C}$; a 4- $\mathrm{mm}$ to 6-mm deviation was grade $\mathrm{D}$; and a $>6-\mathrm{mm}$ deviation was grade E. Breach direction (superior, lateral, inferior, or medial) was also recorded. The pedicle screw position was graded using immediately postoperative CT images obtained in the coronal, axial, and sagittal planes. The grades were assigned by one author (a surgeon other than the operators) at each center.

\section{Robotic Procedure}

\section{CT-to-Fluoroscopy Registration}

This registration method requires the patient to undergo a preoperative thin-cut $(1 \mathrm{~mm}) \mathrm{CT}$ scan that is uploaded to the robotic software to plan the optimal diameter, size, and, most importantly, the trajectory of the screw in all 3 planes (coronal, sagittal, and axial).

After the patient is positioned on the operating room table, a Schanz pin is inserted into the posterior superior iliac spine. The surgical arm is mounted to the operating table via a bed frame adapter and positioned to the patient by way of a bone mount bridge. After this, anterior-toposterior and oblique-to-lateral fluoroscopic radiographs are taken and registered to the robot for intraoperative planning. After forming an intraoperative plan, the cannulated robotic arm moves toward the targeted entry site, after which a drill guide is placed through the cannula and mounted on the targeted anatomy. Following this, a drill is used to create a pilot hole, and a K-wire is placed into the bone. Once the K-wire is securely inserted, the hole is tapped by a ball-tipped probe to ensure no breach occurs and, secondarily, to confirm the screw length. After this, the screw is inserted over the K-wire.

\section{Scan-and-Plan Registration}

This registration method requires an intraoperative 3D CT scan using the O-arm with an array attached to the robotic arm that allows for real-time intraoperative planning without the need for a preoperative thin-cut CT scan and intraoperative fluoroscopic images like the CT-to-fluoroscopy method described above.

After the patient is placed prone on the operating room table with the bed frame adapter secured to the caudal aspect of the bed where the surgical arm is attached, a Schanz pin is drilled into the right posterior superior iliac spine, and the bone mount bridge is attached to the surgical arm. Then, the Stealth reference frame (Medtronic) is placed in the left posterior superior iliac spine. Following this, it is essential to minimize glare by placing a blue tow$\mathrm{el}$ in the region of interest (ROI). The surgical arm has a built-in linear optic camera that performs a 3-define scan over the ROI of the surface environment to delineate the work volume. After this, a reference stylus is placed over the vertebral body within the ROI to help approximate the location, and a star marker is connected to the surgical arm and positioned to the ROI. Next, the O-arm is brought 
in to obtain an intraoperative CT scan; it is important that the scan captures all 4 fiducials on the O-arm's star marker (fiducialized array). After the scan is completed, it is uploaded to the Mazor workstation to plan the screw trajectory and size. Once this portion is complete, the array is removed from the surgical arm and the arm is sent to the first entry point. The remaining steps for inserting the screw are similar to those described above.

\section{Statistical Analysis}

A descriptive analysis was performed. Distribution of the data was assessed using the Shapiro-Wilk test. Continuous data with normal distribution are presented as mean and standard deviation. We used median and IQR for data with a skewed distribution. Categorical variables are presented as percentages and proportions. The independent t-test was used to compare means for continuous data, and the chi-square test was used to compare categorical variables. IBM SPSS Statistics version 25 (IBM Corp.) was used for statistical analysis; $\mathrm{p}<0.05$ was considered significant.

\section{Results \\ Demographics}

Among the 126 patients in the scan-and-plan registration group, $78(61.9 \%)$ were women, with a mean patient age of $60.3 \pm 11.8$ years and a mean BMI of $30.0 \pm 4.9$ $\mathrm{kg} / \mathrm{m}^{2}$, while 58 patients $(46 \%)$ had a smoking history and $46(36.5 \%)$ had a history of previous spine surgery. Among the 142 patients in the CT-to-fluoroscopy registration group, $64(45.1 \%)$ were women, with a mean patient age of $57.1 \pm 14.1$ years and a mean BMI of $28.0 \pm 5.2 \mathrm{~kg} /$ $\mathrm{m}^{2}$, while 90 patients $(63.4 \%)$ had a smoking history, and $26(18.3 \%)$ had undergone previous spine surgery. Demographic data are provided in Table 1.

\section{CT-to-Fluoroscopy Versus Scan-and-Plan Registration}

When comparing the 2 different registration techniques, significant results were observed with regard to fluoroscopy time, with scan-and-plan registration exposing the patient to less overall fluoroscopy time $(\mathrm{p}<0.001)$; however, it exposed them to nonsignificantly more fluoroscopy time per screw $(p=0.3)$. Accuracy rates were not significantly different between the registration methods ( $\mathrm{p}$ $=0.17)$. Estimated blood loss $(\mathrm{EBL})(\mathrm{p}<0.001)$ and the incidence of dural tears $(\mathrm{p}<0.001)$ were also significantly less in the scan-and-plan group; however, these variables cannot be directly related to the registration method that was used. The operative time was significantly longer in the CT-to-fluoroscopy group ( $\mathrm{p}<0.001)$, although this was likely not related to the registration method. Comparative data between the 2 registration methods, and operative data, are provided in Tables 1 and 2 and Fig. 1.

\section{Accuracy of Scan-and-Plan Versus CT-to-Fluoroscopy Registration}

A total of 450 screws were inserted across 266 spinal levels (mean $1.7 \pm 1.1$ screws/level) in the scan-and-plan group. On review of immediately postoperative CT scans,
TABLE 1. Demographic and baseline clinical details

\begin{tabular}{|c|c|c|c|}
\hline Variables & $\begin{array}{l}\text { Scan-and-Plan } \\
\text { Group }\end{array}$ & $\begin{array}{l}\text { T-to-Fluoroscopy } \\
\text { Group }\end{array}$ & p Value \\
\hline No. of pts & 126 & 142 & \\
\hline \multicolumn{4}{|l|}{ Sex } \\
\hline M & $48(38.1)$ & $78(54.9)$ & \multirow{2}{*}{0.005} \\
\hline $\mathrm{F}$ & $78(61.9)$ & $64(45.1)$ & \\
\hline Mean age, yrs & $60.3 \pm 11.8$ & $57.1 \pm 14.1$ & 0.025 \\
\hline Mean BMl, kg/m² & $30 \pm 4.93$ & $28.0 \pm 5.2$ & 0.002 \\
\hline Current or prior smoker & $58(46.0)$ & $90(63.4)$ & 0.006 \\
\hline Previous spinal op & $46(36.5)$ & $26(18.3)$ & 0.001 \\
\hline Diabetes mellitus & $24(19.0)$ & $17(12.0)$ & 0.10 \\
\hline \multicolumn{4}{|l|}{ ASA class } \\
\hline 1 & $2(1.6)$ & $9(6.3)$ & \multirow{3}{*}{0.03} \\
\hline 2 & $72(57.1)$ & $92(64.8)$ & \\
\hline 3 & $52(41.3)$ & $41(28.9)$ & \\
\hline
\end{tabular}

Pts $=$ patients.

Values are presented as the number of patients (\%) or mean \pm SD unless indicated otherwise. Boldface type indicates statistical significance.

446 screws $(99.1 \%)$ were determined to have been placed with grade A accuracy and $4(0.9 \%)$ with grade B accuracy. No screws in this group were replaced intraoperatively. In the CT-to-fluoroscopy group, 574 screws were inserted across 280 spinal levels (mean $2.05 \pm 1.7$ screws/ level). On review of immediately postoperative CT scans, $563(98.1 \%)$ pedicle screws were determined to have been placed with grade A accuracy and $11(1.9 \%)$ with grade $\mathrm{B}$ accuracy on initial $\mathrm{O}$-arm spin, and all 11 were then replaced to grade A accuracy. Postoperatively, 3 patients (2.1\%) in the CT-to-fluoroscopy group had screw-related neurological deficits compared with none in the scan-andplan group.

\section{Discussion}

Pedicle screws have historically been placed using the freehand technique, which relies on anatomical landmarks, or by using the $2 \mathrm{D}$ or $3 \mathrm{D}$ fluoroscopy method as a means for navigation. More recently, surgeons have started using computer-assisted surgery for pedicle screw insertion in which surgeons manually direct screws through the 3D vision capabilities that the computer software provides to increase accuracy rates. ${ }^{3,11,12}$ Robotic guidance allows surgeons to drill the pilot hole and dock the cannula directly to the bone, which eliminates 4 of 6 degrees of freedom of the human arm, allowing for more accurate placement of the screw?

\section{CT-to-Fluoroscopy Registration}

As mentioned previously, CT-to-fluoroscopy registration uses a preoperative CT scan that is merged with an intraoperative fluoroscopy scan-and-plan pedicle screw placement. Previous studies using this registration method have shown high screw placement accuracy rates ranging from $85 \%$ to $99 \%,{ }^{5,13-16}$ although older-generation platforms were used for these studies. Hu et al. reported 
TABLE 2. Operative and perioperative details

\begin{tabular}{|c|c|c|c|}
\hline Variables & $\begin{array}{l}\text { Scan-\&-Plan } \\
\text { Group }(n=126)\end{array}$ & $\begin{array}{l}\text { CT-to-Fluoroscopy } \\
\text { Group }(n=142)\end{array}$ & $p$ Value \\
\hline No. of screws placed & 450 & 574 & \\
\hline \multicolumn{4}{|l|}{ Procedure } \\
\hline TLIF/PLIF & 14 (11.1) & $106(74.6)$ & \multirow{6}{*}{$<0.001$} \\
\hline OLIF & $29(23.0)$ & $16(11.3)$ & \\
\hline ALIF & $19(15.1)$ & $1(0.7)$ & \\
\hline XLIF & $27(21.4)$ & 0 & \\
\hline MIDLIF & $29(23.0)$ & 0 & \\
\hline Other & $6(4.8)$ & $19(13.3)$ & \\
\hline $\begin{array}{l}\text { No. of pts w/ percutane- } \\
\text { ous screws }\end{array}$ & 77 (61.1) & 0 & $<0.001$ \\
\hline \multicolumn{4}{|l|}{ Operated levels } \\
\hline Single level & $18(14.3)$ & $34(23.9)$ & \multirow{2}{*}{0.05} \\
\hline Multiple levels & $108(85.7)$ & $108(76.0)$ & \\
\hline $\mathrm{L} 1$ & $3(2.4)$ & $2(1.4)$ & \multirow{6}{*}{$<0.001$} \\
\hline L2 & $11(8.7)$ & $5(3.5)$ & \\
\hline L3 & $41(32.5)$ & $18(12.6)$ & \\
\hline L4 & $94(74.6)$ & $84(59.1)$ & \\
\hline L5 & $98(77.8)$ & $105(73.9)$ & \\
\hline S1 & $19(15.1)$ & $66(46.5)$ & \\
\hline \multicolumn{4}{|l|}{ Periop data } \\
\hline Mean op time, mins & $207 \pm 80$ & $296.3 \pm 134.4$ & $<0.001$ \\
\hline Mean EBL, ml & $77.7 \pm 119$ & $428.1 \pm 469$ & $<0.001$ \\
\hline $\begin{array}{l}\text { Robot abandoned due } \\
\text { to registration error }\end{array}$ & $4(3.2)$ & $8(5.6)$ & 0.33 \\
\hline $\begin{array}{l}\text { Mean fluoroscopy } \\
\text { time, sec }\end{array}$ & $36.9 \pm 36.7$ & $57.8 \pm 27.1$ & $<0.001$ \\
\hline $\begin{array}{l}\text { Mean fluoroscopy } \\
\text { time/screw, sec }\end{array}$ & $12 \pm 13$ & $11.1 \pm 7$ & 0.3 \\
\hline \multicolumn{4}{|l|}{$\begin{array}{l}\text { Gertzbein-Robbins } \\
\text { grade* }^{*}\end{array}$} \\
\hline A & $446(99.1)$ & $563(98.1)$ & 0.17 \\
\hline B & $4(0.9)$ & $11(1.9)$ & 0.17 \\
\hline \multicolumn{4}{|l|}{ Complications } \\
\hline Dural tear & $1(0.8)$ & $19(13.4)$ & $<0.001$ \\
\hline $\begin{array}{l}\text { Postop neurological } \\
\text { deficit }\end{array}$ & 0 & $2(1.4) \dagger$ & 0.2 \\
\hline $\begin{array}{l}\text { Urinary retention } \\
\text { (transient) }\end{array}$ & $1(0.8)$ & $1(0.7)$ & 0.9 \\
\hline $\begin{array}{l}\text { Surgical site } \\
\text { infection }\end{array}$ & $1(0.8)$ & $4(2.8)$ & 0.2 \\
\hline Wound dehiscence & $1(0.8)$ & 0 & 0.3 \\
\hline
\end{tabular}

ALIF = anterior lumbar interbody fusion; MIDLIF = midline lumbar interbody fusion; OLIF = oblique lateral interbody fusion; PLIF = posterior lumbar interbody fusion; TLIF = transforaminal lumbar interbody fusion; XLIF = extreme lateral interbody fusion.

Values are presented as the number of patients (\%) or mean \pm SD unless indicated otherwise. Boldface type indicates statistical significance.

* Values are presented as the number of screws (\%).

$\dagger$ One case of weakness after the patient experienced a fall and another case of worsening lower-extremity weakness. an accuracy rate of $98.9 \%$ after studying 960 screw insertions; inaccuracy was attributed to misplacement and skiving of screws. ${ }^{5}$ Those authors did not report radiation dose or operative time. Keric et al. studied 2067 screw insertions and reported an accuracy rate of $96.7 \%$ using the Renaissance robot (Mazor) with a 258.7-minute operative time, which included registration of the robot, screw implantation, and the decompression and fusion procedures that were performed in conjunction with the screw insertions; however, radiation exposure was not included in the study variables..$^{15}$ Pechlivanis et al. used the Mazor SpineAssist and reported an accuracy rate of $91.7 \% .^{13}$ Ringel et al. studied 146 screw placements using the SpineAssist and reported an accuracy rate of $85 \%$ with an operative time of 151 minutes with lumbar decompression of the neural elements and 95 minutes without decompression. ${ }^{14}$ In that study, radiation exposure to the patient during planning was $411.6 \mathrm{mGy}$. Onen et al. measured screw accuracy across 136 screws using the Renaissance robot and reported an accuracy rate of $98.5 \%$, with a mean operative time of 59.2 minutes and mean fluoroscopy exposure time of 1.3 seconds. ${ }^{16}$ Both Renaissance and SpineAssist involve preoperative planning using CT-to-fluoroscopy registration. In 142 patients with 574 screw placements, we achieved an accuracy rate of approximately $98.1 \%$ (grade A, Gertzbein-Robbins scale), which is commensurate with those previously reported, and with a comparable fluoroscopy time as well.

\section{Scan-and-Plan Registration}

The scan-and-plan registration method uses an intraoperative $\mathrm{CT}$ scan acquired using the $\mathrm{O}$-arm while the patient is positioned on the operating room table and real-time imaging and anatomy to create a pedicle screw plan for each patient. Literature evaluating scan-and-plan registration is scarce. Khan et al. published the first study showing the scan-and-plan method as a viable option. ${ }^{7}$ Again, it is worth noting that among the 20 patients included in that study, 12 underwent scan-and-plan registration and 8 underwent CT-to-fluoroscopy registration. Accuracy in the CT-to-fluoroscopy group was $100 \%$, whereas in the scanand-plan group, it was $97.7 \%$; however, this difference was not statistically significant. Operative time was not compared between the 2 registration techniques; however, the authors did report a variable robot time, which they defined as the time from when the robot moved to the specified trajectory to when the screw was inserted. Robot time was less with CT-to-fluoroscopy registration than scanand-plan registration, although this was not statistically significant. Patients in the scan-and-plan group had significantly less overall radiation exposure compared with the CT-to-fluoroscopy group; however, this became nonsignificant when calculated per screw. This is attributed to the longer fusion segments per patient in the CT-to-fluoroscopy group. Khan et al. used scan-and-plan registration in another study consisting of 42 patients receiving 158 screw placements; accuracy was $99.3 \%$ with a Ravi grade of I, with 1 screw placement being Ravi grade II, although that is also considered clinically accurate. ${ }^{6}$ In that study, the mean operative time was 153.9 minutes and mean radiation dose was $58.5 \mathrm{mGy}$. Khan et al. also studied 22 

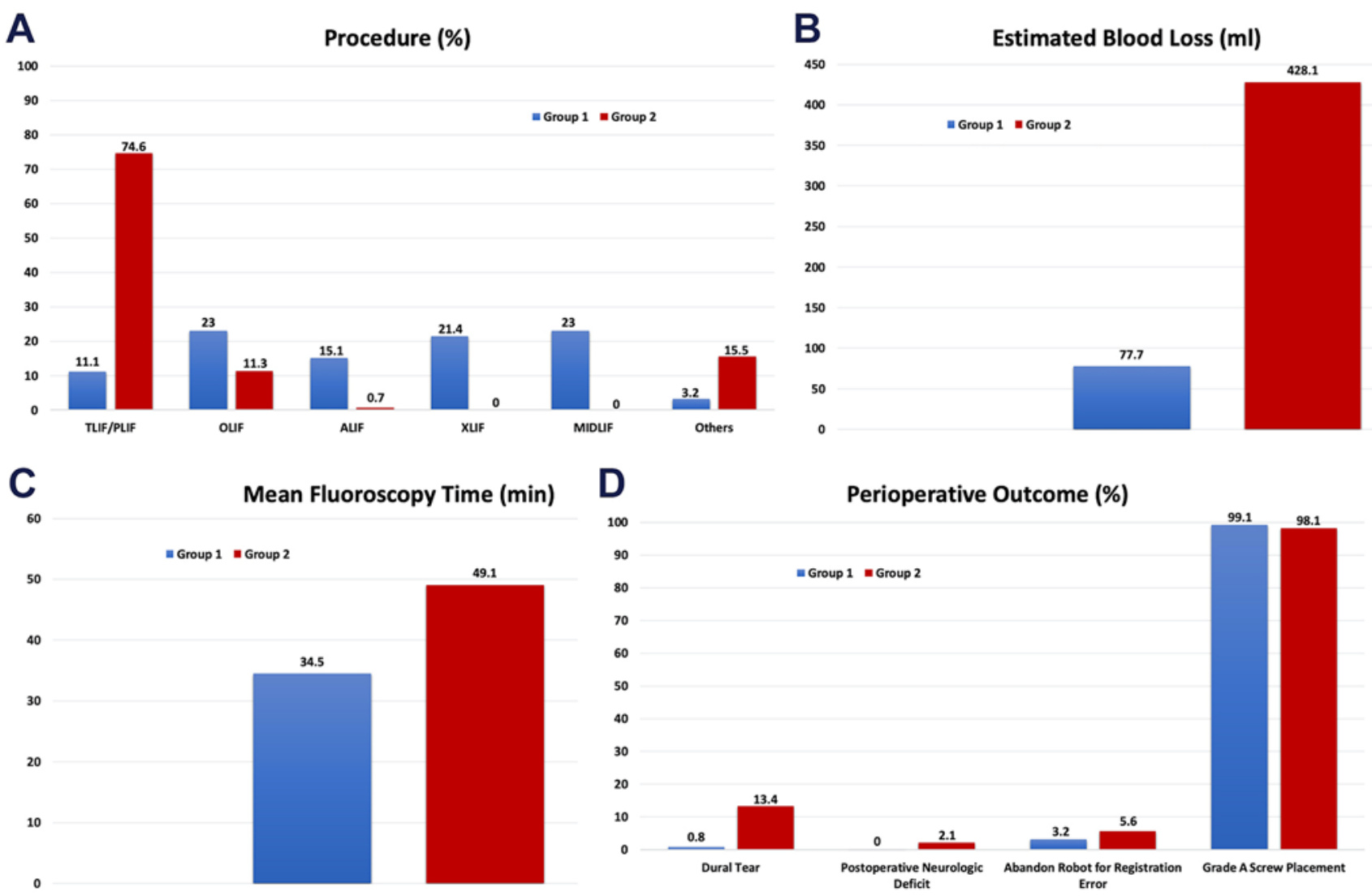

FIG. 1. Bar graphs comparing operative and perioperative data in the scan-and-plan group (blue, group 1) with those in the CT-tofluoroscopy group (red, group 2). A: Procedure. B: Mean EBL. C: Mean fluoroscopy time. D: Perioperative outcome. ALIF = anterior lumbar interbody fusion; MIDLIF = midline lumbar interbody fusion; OLIF = oblique lateral interbody fusion; PLIF = posterior lumbar interbody fusion; TLIF = transforaminal lumbar interbody fusion; XLIF = extreme lateral interbody fusion.

patients in whom scan-and-plan registration was used for pedicle screw insertion through cortical bone trajectories. ${ }^{8}$ Accuracy was $100 \%$ (Ravi grade I); the mean operative time was 180.2 minutes, the mean fluoroscopy time was 10.1 seconds, and the mean radiation dose was $38.1 \mathrm{mGy}$. The current study included 126 patients in whom scanand-plan registration was used and $99.1 \%$ accuracy was demonstrated using the Gertzbein-Robbins scale, providing further evidence that this method of registration is safe and accurate. Furthermore, the remaining $0.9 \%$ had grade B accuracy, which is not considered clinically significant, so a decision was made not to revise these screws in the scan-and-plan group.

\section{Surgical Considerations}

Surgeon preference in registration technique is the single most important factor in operating room workflow. However, certain considerations should be made, because CT-to-fluoroscopy registration requires the patient to undergo an additional preoperative CT scan for registration followed by additional fluoroscopic images intraoperatively, thus exposing the patient to more radiation, as demonstrated in a previous study. ${ }^{7}$ Furthermore, in terms of the nuances to registration when CT-to-fluoroscopy is used, each vertebral body is registered independently in seg- mentation based on the preoperative scan. However, with the scan-and-plan method, the $\mathrm{O}$-arm spin is a nonsegmented global registration scan obtained with the patient already positioned on the operating room table, which may account for the slight increase in accuracy of screw placement in our study, although it is important to note that these results were not statistically significant. Use of the O-arm intraoperatively with scan-and-plan registration does add to the overall operative time in a general sense; however, in our study, scan-and-plan registration required less operative time overall.

\section{Limitations}

Our study has the inherent limitations of a retrospective review design using data from 2 different surgeons at 2 different centers. The primary outcome of this study was to examine radiographic outcomes in terms of the accuracy of screw insertion. Ideally, an independent radiologist would have reviewed all CT scans. Furthermore, using data from 2 different surgeons is not an optimal approach, as certain surgeons have their own nuances in the operating room that could skew the results of screw placement. The radiation dose was not available for all patients, so fluoroscopy time was used as a substitute for that variable; ideally, the radiation dose in milligrays is a far 
superior representation. Blood loss was far greater in the CT-to-fluoroscopy group because those cases were mostly performed through an open technique with longer fusion segments, while the cases in the scan-and-plan group were performed through a minimally invasive technique. Finally, due to the retrospective nature of this study, there was deficiency in the patient-reported outcome measures, so we could not include those data in our analysis.

\section{Conclusions}

We present what is, to our knowledge, the first analysis comparing 2 different radiographic registration techniques with the newest-generation robotic technology available in spine surgery. Both the CT-to-fluoroscopy and scan-andplan registration methods demonstrated high accuracy as previously reported in the literature, although in terms of CT-to-fluoroscopy, the previously published literature used older-generation platforms. Furthermore, scan-andplan registration exposed the patient to a similar fluoroscopy exposure. This study lays the groundwork for future studies comparing different registration techniques using robotic spine technology in the operating room. Future studies are warranted to verify the results of the presented study.

\section{Acknowledgments}

We thank Paul H. Dressel, BFA, for formatting the illustration and Debra J. Zimmer for editorial assistance.

\section{References}

1. Faraj AA, Webb JK. Early complications of spinal pedicle screw. Eur Spine J. 1997;6(5):324-326.

2. Solomiichuk V, Fleischhammer J, Molliqaj G, et al. Robotic versus fluoroscopy-guided pedicle screw insertion for metastatic spinal disease: a matched-cohort comparison. Neurosurg Focus. 2017;42(5):E13.

3. Kosmopoulos V, Schizas C. Pedicle screw placement accuracy: a meta-analysis. Spine (Phila Pa 1976). 2007;32(3): E111-E120.

4. Castro WH, Halm H, Jerosch J, Malms J, Steinbeck J, Blasius S. Accuracy of pedicle screw placement in lumbar vertebrae. Spine (Phila Pa 1976). 1996;21(11):1320-1324.

5. Hu X, Ohnmeiss DD, Lieberman IH. Robotic-assisted pedicle screw placement: lessons learned from the first 102 patients. Eur Spine J. 2013;22(3):661-666.

6. Khan A, Meyers JE, Yavorek S, et al. Comparing next-generation robotic technology with 3-dimensional computed tomography navigation technology for the insertion of posterior pedicle screws. World Neurosurg. 2019;123:e474-e481.

7. Khan A, Meyers JE, Siasios I, Pollina J. Next-generation robotic spine surgery: first report on feasibility, safety, and learning curve. Oper Neurosurg (Hagerstown). 2019;17(1): 61-69.

8. Khan A, Rho K, Mao JZ, et al. Comparing cortical bone trajectories for pedicle screw insertion using robotic guidance and three-dimensional computed tomography navigation. World Neurosurg. 2020;141:e625-e632.
9. von Elm E, Altman DG, Egger M, Pocock SJ, Gøtzsche PC, Vandenbroucke JP. Strengthening the Reporting of Observational Studies in Epidemiology (STROBE) statement: guidelines for reporting observational studies. BMJ. 2007; 335(7624):806-808.

10. Gertzbein SD, Robbins SE. Accuracy of pedicular screw placement in vivo. Spine (Phila Pa 1976). 1990;15(1):11-14.

11. Verma R, Krishan S, Haendlmayer K, Mohsen A. Functional outcome of computer-assisted spinal pedicle screw placement: a systematic review and meta-analysis of 23 studies including 5,992 pedicle screws. Eur Spine J. 2010;19(3):370375 .

12. Aoude AA, Fortin M, Figueiredo R, Jarzem P, Ouellet J, Weber MH. Methods to determine pedicle screw placement accuracy in spine surgery: a systematic review. Eur Spine $J$. 2015;24(5):990-1004.

13. Pechlivanis I, Kiriyanthan G, Engelhardt M, et al. Percutaneous placement of pedicle screws in the lumbar spine using a bone mounted miniature robotic system: first experiences and accuracy of screw placement. Spine (Phila Pa 1976). 2009; 34(4):392-398.

14. Ringel F, Stuier C, Reinke A, et al. Accuracy of robot-assisted placement of lumbar and sacral pedicle screws: a prospective randomized comparison to conventional freehand screw implantation. Spine (Phila Pa 1976). 2012;37(8):E496-E501.

15. Keric N, Doenitz C, Haj A, et al. Evaluation of robot-guided minimally invasive implantation of 2067 pedicle screws. Neurosurg Focus. 2017;42(5):E11.

16. Onen MR, Simsek M, Naderi S. Robotic spine surgery: a preliminary report. Turk Neurosurg. 2014;24(4):512-518.

\section{Disclosures}

Dr. Khan received a research grant from the Scoliosis Research Society to study scoliosis in Chiari patients. Ms. Mao receives research funding from AO Spine North America (AOSNA) for works to advance 3D printing. Dr. Lehman receives funding from the Department of Defense and is a consultant for and receives royalties from Medtronic. Dr. Mullin receives research funding from AO Spine North America (AOSNA) and the Research Committee Award \#87639; and from Medtronic External Research Program Health Professionals, ERP ID\#2020-12271. Dr. Pollina is a consultant and involved with surgical training for Medtronic. He serves as a consultant for and receives royalties from ATEC Spine.

\section{Author Contributions}

Conception and design: Pollina, Khan. Acquisition of data: Khan, Lee. Analysis and interpretation of data: all authors. Drafting the article: Khan, Soliman, Lee. Critically revising the article: all authors. Reviewed submitted version of manuscript: all authors. Approved the final version of the manuscript on behalf of all authors: Pollina. Study supervision: Pollina.

\section{Correspondence}

John Pollina: University at Buffalo, NY.jpollina@ubns.com. 